\title{
NAV2729 in combination with Imatinib promote apoptosis in chronic myeloid leukemia cell line, K562
}

Hajar Mardani ( $\sim$ hajarmardani61@gmail.com )

Kerman University of Medical Sciences https://orcid.org/0000-0003-2403-4929

\section{Zahra Shakerinia}

Kerman University of Medical Sciences

Roohollah Mirzaee Khalilabadi

Kerman University of Medical Sciences

Ahmad Fatemi

Gerash University of Medical Sciences

Alireza Farsinejad

Kerman University of Medical Sciences

\section{Research Article}

Keywords: Chronic Myeloid Leukemia, K562 Cells, Extracellular Vesicles, apoptosis

Posted Date: June 9th, 2022

DOI: https://doi.org/10.21203/rs.3.rs-1277762/v2

License: (c) (i) This work is licensed under a Creative Commons Attribution 4.0 International License. Read Full License 


\section{Abstract}

Background: Chronic myeloid leukemia (CML) is a hematologic malignancy that affect hematopoietic stem cells. CML therapy prevents the disease from spreading to more advanced stages and improve quality of life. Imatinib as the first line treatment for $\mathrm{CML}$ is associated with side effects and resistant. Tumor derived extracellular vesicles (TMVs) play an important role in the progression of the disease. Inhibiting the release of TMVs, helps to inhibit leukemia. Our purpose is to investigate the apoptotic effect of NAV2729 in combination with Imatinib on CML cell line, K562.

Methods and Results: After treatment of K562 cells with NAV2729, Imatinib and both, viability of cells were measured after 24h, 48h. Metabolic activity, changes in Bax and Bcl-2 expressions, size and concentration of EVs were evaluated after 48h treatment of K562 cells with NAV2729, Imatinib and both. Trypan blue assay indicated, decremented live cells percentage. MTT assay showed reduction in metabolic activity when both of the drugs were used. Reduction in BCL-2 and BAX expressions was observed. EVs count release from K562 cells were decreased in the combination of drugs.

Conclusion: study result showed combined treatment of NAV2729 and imatinib synergistically inhibit K562 cell proliferation and enhances the therapeutic effect of imatinib.

\section{Introduction}

Chronic myeloid leukemia (CML) is a leukemia that occurs in the hematopoietic stem cells associated with a chromosomal abnormality, $t$ (9:22) reciprocal translocation producing BCR-ABL1 tyrosine kinase; leading uncontrolled phosphorylation and activation of downstream signal pathways. As a result, this shift alters cell binding, inhibits apoptosis, stops differentiation and breaks down key proteins, and creates a disease phenotype.[1] Imatinib is the first tyrosine kinase inhibitor received FDA approval for treatment of CML patients. It is a competitive inhibitor, bounds to the ATP-binding region in BCR-ABL1 oncogene, inhibiting the phosphorylation of cell signaling pathway proteins. [2]Thus, it inhibits proliferation and induces apoptosis in CML cancer cells. Despite the choice of Imatinib as the first line of treatment, it is associated with side effects and also there is possibility of disease recurrence or resistance in some patient. [3]

Extracellular vesicles (EVs) are small membrane vesicles that originate from the cells. These vesicles are surrounded by phospholipid bilayers, and the intracellular components enclosed by this membrane are released into the environment and into body fluids, including Blood, urine, ascites, etc. are found. $[4,5]$ After release, they fuse with other cells and then transfer their cytoplasmic contents to them, affecting the target cell.[6] Extracellular vesicles that are released from tumor cells in vivo and in the laboratory are called tumor-derived extracellular vesicles (TEVs) [7, 8]. Also, from 2008 onwards, a term called Oncosomes was expressed and studied. These extracellular vesicles with a diameter of about 1-10 micrometers are derived from tumor cells $[9,10]$. The roles of TEV include: transmits growth factor receptors, enhanced cell motility, induction of angiogenesis, escape from immune system detection and 
drug resistance [11] and increase tumor invasion, migration, proliferation[12, 13] and development of signal response. It also modulates the microenvironment and provides a suitable environment for tumor cell growth and survival $[14,15]$ one of the most important thing in cancer treatment is keeping the drug in cancer cells to improve the effect of the drug. The secretion of TEV from cancer cells causes drug efflux and thus reduce the drug's effect on Tumor cells [16] it has been shown that Inhibition of EV shedding increases drug retain and sensitize tumor to chemotherapy [17] The mechanism of EV production is influenced by various factors, one of which is ARF6. ARF6 is part of the ARF family, which is a GTPbinding, Ras-dependent protein [11].ARF6 molecules are required for the formation and release of EVs.[18] This study was performed to assay the effect of extracellular vesicle inhibitor on k562, human chronic myeloid cell. The combination of Imatinib with NAV2729, which is an ARF6 inhibitor (ARF6 is one of the essential factors for the production and secretion of vesicles) it placed.

\section{Material And Method}

\subsection{Cell culture and Drug treatment:}

K562 cell lines, were cultured in RPMI 1640 medium with 10\% fetal bovine serum (FBS) and 100 units $/ \mathrm{ml}$ penicillin, also $100 \mu \mathrm{g} / \mathrm{ml}$ streptomycin in a humidified atmosphere at $37^{\circ} \mathrm{C}$ that consist of $5 \% \mathrm{CO} 2$.

\subsection{Measurement of cell viability by trypan blue exclusion assay:}

To investigate the effect of NAV2729, Imatinib and Combination of them on cell viability, K562 cells were tested to trypan blue exclusion assay. Cells were cultured in a cell culture plate of 6-well with a density of cells per well and treated with different concentrations of NAV2729 and Imatinib and a combination them. After incubation at 24 and 48 hours, each cell suspension was mixed with $0.4 \%$ trypan blue solution in a ratio of 1:1 after 1_2 minutes of incubation at room temperature, the mixture was examined on a slide on a hemocytometer and under a microscope. Live cells unlike dead cells, are clear then were counted and the survival rate was calculated based on the following formula: (number of live cells counted/total number of cells counted) $\times 100$.

\subsection{Metabolic activity measured by MTT assay:}

The effect of various concentrations of NAV2729, Imatinib and Combination of them on metabolic activity of K562 cells was assayed by the MTT colorimetric method. Cells were cultured in 96 cell culture plate with a density of cells per well and treated with different concentrations of NAV2729, Imatinib and a combination of them. After 48 hours of incubation, the medium was discarded and cells were incubated with the MTT solution ( $5 \mathrm{mg} / \mathrm{ml}$ in PBS) for $4 \mathrm{~h}$ at $37^{\circ} \mathrm{C}$. The resulting formazan crystals were solubilized by addition of $100 \mathrm{ml}$ dimethyl sulfoxide (DMSO) at each well, and the absorbance was measured at 570 nm by ELISA reader.

\subsection{Apoptosis assay:}


Cell death of k562 cells was assayed by apoptosis assay. Cells were seeded into 6-well cell culture plates also Cells were treated on NAV2729, Imatinib and Combination of them. After 48h incubation the cells were collected, Washed with FBS and stained using FITC Annexin-V Apoptosis Detection Kit II (BD Biosciences, USA), according to the manufacturer's instructions. The percentage of apoptotic cells was quantified using Becton-Dickinson FACS. Annexin-V positive and PI-negative cells were considered to be in early apoptotic phase, and cells having positive staining both for Annexin-V and PI were deemed to undergo late apoptosis or necrosis.

\subsection{RNA isolation and preparation of cDNA:}

K562 cells were treated with NAV2729, Imatinib and combination of them, RNA K562 cells were isolated after $48 \mathrm{~h}$ incubation by using TriPure Isolation Reagent according to the instructions of the manufacturer (Roche). Reverse transcription (RT) reaction was performed using the RevertAid First Strand complementary DNA (cDNA) Synthesis kit from Yekta tajhize. A reaction contained 9- $\mu$ l nuclease-free water, $1 \mu$ l Random Hexamer Primer, $5 \mu$ l $5 \times$ Reaction Buffer, $2 \mu$ dNTP Mix (10 mM), $1 \mu$ l RiboLock RNase Inhibitor $(20 \mathrm{U} / \mu \mathrm{l})$, and $1 \mu \mathrm{l}$ RevertAid M-MuLV Reverse Transcriptase $(200 \mathrm{U} / \mu \mathrm{l})$ as a master mix, and $2 \mu \mathrm{l}$ of total RNA ( $1 \mu \mathrm{g}$ per reaction) was added prior to reaction start. for the reverse transcription were incubated $5 \mathrm{~min}$ at $25^{\circ} \mathrm{C}$ followed by $60 \mathrm{~min}$ at $42{ }^{\circ} \mathrm{C}$ and The reaction was terminated by heating at $70{ }^{\circ} \mathrm{C}$ for $5 \mathrm{~min}$.

\subsection{Quantitative real-time PCR:}

Real-time PCR was performed using 7.5 $\mu$ l of RealQ Plus 2×Master Mix Green (Amplicon), $1.5 \mu$ l of the cDNA product, $1 \mu \mathrm{l}$ of each of forward and reverse primers, and $4 \mu \mathrm{L}$ of nuclease-free water in a total volume of $15 \mu \mathrm{l}$. Thermal cycling conditions included an initial activation step at $95^{\circ} \mathrm{C}$ for $15 \mathrm{~min}$ followed by 40 cycles, including a denaturation step at $95^{\circ} \mathrm{C}$ for $15 \mathrm{~s}$ and a combined annealing/elongation step at $60{ }^{\circ} \mathrm{C}$ for $60 \mathrm{~s}$. The reaction took place in the Rotor Gene 6000 Real-time PCR System (Corbett Research). A melting curve analysis was performed to verify the specificity of the products. The fold induction or repression was measured relative to the control and calculated after

adjusting for the GAPDH reference gene using the comparative $\mathrm{Ct}\left(2^{-\Delta \Delta C T}\right)$ method. Primer sequences are available upon request.

\subsection{Microvesicles isolation:}

The cell contents in the treated and control groups centrifuged at room temperature at 4000 RPM for 10 minutes to remove the cells. The supernatant was slowly transferred to another tube. This step was repeated once more and then the supernatant was transferred to the microtube and centrifuged 30 minutes at 20,000 $\mathrm{g}$ to isolate the micrvesicles. Finally the supernatant was removed and the microvesicles precipitate was suspended in $1 \mathrm{ml}$ of RPMI1640 suspension. 


\subsection{Determining the size and concentration of isolated microvesicles:}

ZETA SIZER MALVERN, which is based on DLS, was used to determine the size and concentration. In this system, laser light with wavelength is used. In this system, by shining a laser light with a wavelength of $633 \mathrm{~nm}$ to the suspension containing microvesicles as well as RPMI1640 medium as a control sample, scattered lights were identified and the size and concentration of microvesicles and particles in the software were recorded.

\section{Statistical analysis:}

Two-tailed t-test was used to determine if there is a significant difference between experimental variable. A P-value $<0.05$ was considered statistically significant.

\section{Results}

\subsection{Nav2729 in combined with Imatinib reduced cell viability in k562 cells}

24 and 48 hours after treatment of K562 cells with NAV2729 and Imatinib drugs separately and combination of them, cell viability was evaluated by trypan blue dye excretion method. As shown in Fig1. Cell viability was significantly reduced after exposure to NAV2729 in a dose- and time-dependent trend. Decreased cell viability was observed after treatment with Imatinib in a dose-dependent manner. At combined treatment, cell viability was further reduced than when the cells were treated separately with drugs.

\subsection{Reduction of cellular metabolic activity by MTT colorimetric test after treatment of K562 cells with NAV2729, Imatinib and combination of them}

K562 cells were treated with different concentrations of NAV2729 and Imatinib, 48 hours after treatment, MTT staining test was used to evaluate the cellular metabolic activity. Dose-dependent decrease in cellular metabolic activity is observed in cells treated with NAV2729 and Imatinib. The metabolic activity of K562 cells in the combinations of the two drugs was significantly lower than the separate doses (Fig2).

\subsection{NAV2729, Imatinib and combination of them did not affect the metabolic activity of normal human PBMCs}

In order to evaluate the cytotoxic effect of NAV2729 on normal cells, after isolating human PBMCs and treating them with $5 \mu \mathrm{M}$ concentration using the MTT colorimetric test, cellular metabolic activity was assessed within 48 hours. The results indicate that NAV2729 does not have a significant cytotoxic effect on normal human cells (PBMC). 


\subsection{Altered expression of apoptosis-related genes by combined and separate treatment of NAV2729 and Imatinib in K562 cells}

In order to evaluate the expression of apoptosis-related genes, K562 cells treated with a combined of NAV2729 $(5 \mu M)+$ Imatinib $(4 \mu M)$, Imatinib $(5 \mu M)$ and Imatinib $(4 \mu M)$ were used separately. According to figure 3(a), our data showed that the expression of Bcl-2 anti-apoptotic gene did not change significantly for induction of apoptosis by NAV2729 and Imatinib, while the combined treatment of drugs resulted in a significant reduction in Bcl-2 expression compared to separate doses. It has been medicated ( $p$-value $<0.05)$. As shown in figure $3(\mathrm{~b})$, the expression of Bax pre-apoptotic protein gene was increased during 48-hour treatment of K562 cells by separate doses of NAV2729 and Imatinib, and the combined dose showed decreased gene expression.

\subsection{Imatinib, NAV2729, and a combination of them promote apoptosis in k562 cells:}

To evaluate the cytotoxic effect induced by the combined dose of NAV2729 $(5 \mu \mathrm{M})+$ Imatinib $(4 \mu \mathrm{M})$ on k562 cells, the rate of apoptosis during 48 hours of treatment with these two drugs was evaluated separately and in combination. In this regard, FITC Annexin V Apoptosis Detection Kit II was used. The results shown in the following figure indicate the induction of apoptosis separate and combined doses of Imatinib and NAV2729 on k562 cells. Also AnnexinV-positive and AnnexinV / PI-positive cells, indicating apoptotic cells, in a 48-hour treatment with a combined dose of NAV2729 $(5 \mu \mathrm{M})+$ Imatinib $(4 \mu \mathrm{M})$ relative to cells which were treated by separate doses of these two drugs constitute a higher percentage.

According to the results, the combined dose of NAV2729 $(5 \mu \mathrm{M})+$ Imatinib $(4 \mu \mathrm{M})$ on k562 cells after 48 hours resulted in apoptosis of $62.64 \%$ of the cells compared to the separate doses of NAV2729 (5 $\mu \mathrm{M})$ and Imatinib $(4 \mu \mathrm{M})$, which resulted in $15.64 \%$ and $18.64 \%$ of cells, respectively, and differed more than the control group. This may indicate that the use of a combined dose can help reduce the dose of chemotherapy drugs, which can be seen in the figure below. The increase in apoptosis in the combined dose of two drugs is significantly different from the separate dose of Imatinib.

\subsection{Determination of size and concentration of extracellular vesicles by DLS method}

Using the MALVERN, $633 \mathrm{~nm}$ wavelength, we assess size and concentraton of extracellular vesicles derived from k562 cells treated with imatinib and NAV2729. The results indicated that the amount of vesicles more than $1000 \mathrm{~nm}$ in the NAV2729 drug treatment alone and combined with Imatinib reduced. (Figure 5)

\section{Discussion}

Until 2000, pharmacotherapy for CML was limited to nonspecific agents such as busulfan, hydroxyurea, and interferon-alpha (IFN-a), and allogeneic stem cell transplantation (allo-SCT) is another treatment reduces mortality risks. It has limitations that cannot be used by all patients and there is a possibility of disease recurrence[19,20] CML therapy has changed with the development of tyrosine kinase inhibitors 
(TKIs) that potentially interfere with the interaction between BCR-ABL1 oncoprotein and adenosine triphosphate (ATP) and inhibit malignant clone proliferation[21]. This targeted approach changed the history of CML treatment and improved the 10-year survival rate from about $20 \%$ to $80-90 \%[19,22]$. Imatinib is the first FDA approved TKI for the treatment of patient with CML. It competitively inhibits ATP binding to BCR-ABL1 oncoprotein, which inhibits the phosphorylation of proteins involved in cell signal transduction. It also blocks the Platelet derived growth factor receptor (PDGFR) and tyrosine kinase C$\mathrm{KIT}[2,23]$. However, imatinib causes annoying side effects on quality of life, including weight gain, fatigue, nausea, bone and muscle aches, most of which are mild to moderate. Less than $5 \%-10 \%$ experience increase in creatinine with long-term treatment $[19,24]$.The annual incidence of CML in 20302040 is estimated to be about 35 times higher and also the prevalence of nearly 3 million people worldwide is predicted during these years [19]. Therefore, drug availability, development of long-term side effects, and treatment's costs may place a significant burden on patients and the health care system. Therefore, it is imperative to research on therapeutics that is more effective and also have excellent long term survival. In this context, research on new tyrosine kinase, Combination therapy with other drugs and inhibitors can be reviewed. Such strategies may improve the eradication of minimal residual disease and potentially eliminate the need for unlimited TKI treatment. In this study, the combined use of Imatinib as a tyrosine kinase inhibitor and NAV2729 as an extracellular vesicle inhibitor was investigated. Tumorderived extracellular vesicles have significant effects on disease development and progression. In 2008, Janus Rak first coined the term oncosome [9]. In 2015, Valentina R. Minciacchi et al. Showed that prostate cancer cells release bioactive EVs 1 to 10 microns in diameter, giving them the term large oncosomes, which appear to contain carcinogenic and cancer-specific substances. These studies were the first to show that these non-apoptotic membrane buds can be released from cancer cells in the form of large oncosomes [10,25] Because oncosomes, like MVs, originate from the plasma membrane, some of the pathways involved in the biogenesis of MVs may also play a role in the formation of large oncosomes. For example, ARF6, which is involved in the formation of MV from plasma membranes and is found in MV $[26,27]$ is also highly expressed in large oncosomes [28]. In our study, extracellular vesicles with the diameter of more than $1000 \mathrm{~nm}$ were found both in control samples and separate doses of Imatinib treated samples, while in the sample of NAV2729 treatment alone and also As well as sample treated with combination, the percentage of vesicles with a diameter of more than $1000 \mathrm{~nm}$, were $0 \%$ and $1.1 \%$, respectively, which indicates Inhibitory effect of NAV2729 on EV secretion.

A 2017 study by Uchini S. Kosgodage et al. examined the combined effects of chemotherapy drugs and inhibitors of extracellular vesicles secretion, two inhibitors of extracellular vesicles, Chloramidine and Bisindolylmaleimide, reduced PC3 viable cells, by about $62 \%$ and $59 \%$, respectively[17]. In another study by Samireh jorfi et al. MVs with Decetaxel chemotherapy (DTX) tripled the concentration of chemotherapy in tumor cells [29] Further studies by Pinar Uysal-onganer et al. Indicated that the rate of LN18 cell invasion decreased by decreasing in EV secretion.[30]Decreased cell viability and metabolic activity, indicating inhibition of cell proliferation. In our study, imatinib and NAV2729 did not induce any changes in expression of $\mathrm{Bcl} 2$ gene significantly, but in the combined treatment, a decrease in bcl2 gene expression was observed. Also, Bax gene expression was increased in separate drug therapy, while a 
combined treatment showed a decrease in gene expression. A study by Keeshan et al. in 2001, examined the combination therapy of imatinb with cytotoxic drugs on hypersensitive, drug-resistant cells, it was concluded that the combined use of these two drugs increase the expression of the bax gene and decrease the expression of the bcl 2 gene, which induces apoptosis through the caspase 3 and PRAP pathways and causes gene expression, shift to proapoptotic [31] In 2004, Kawano et al. investigated the effect of histone deacetylase inhibitor in combination with Imatinib on induction of apoptosis. The results of this study showed that Imatinib alone reduced bax and bcl2 and histone deacetylase inhibitor alone did not alter gene expression, whereas in the combination of the two drugs, expression of bax and bcl2 decreased. The decrease in bcl 2 was less than bax. In general, combination therapy increased the induction of apoptosis through caspase-3 degradation and PRAP [32].

\section{Conclusion}

This study indicates the effective role of extracellular vesicle inhibitors in increasing the effect of chemotherapy and the correct and accurate selection of extracellular vesicle inhibitors inhibits the biogenesis of these vesicles. The results of this study in the chronic myeloid leukemia cell line suggest further research into other types of leukemia, which could be a promising strategy to increase the effectiveness of treatment as well as reduce the duration of treatment for cancer patients.

\section{Declarations}

\section{Acknowledgments}

This study has been done as part of the master thesis for Zahra Shakerinia. The authors wish to thanks Kerman University of Medical Sciences for their financial support.

\section{Authors' Contributions:}

Hajar Mardani Valandani, Participated in study design and conception. Zahra Shakerinia, performed experimental work, molecular experiments and RT-qPCR analysis and drafted the paper. Roohollah Mirzaee Khalilabadi Contributed in conception and design. Also contributed in qRT- PCR and cell cycle analysis. Ahmad Fatemi Contributed in interpretation of the data and the conclusion. Alireza farsinejad was responsible for overall supervision and Contributed in revising the paper.

\section{References}

1. A. Bennour, A. Saad, and H. Sennana, "Chronic myeloid leukemia: Relevance of cytogenetic and molecular assays," Critical reviews in oncology/hematology, vol. 97, pp. 263-274, 2016.

2. B. J. Druker and N. B. Lydon, "Lessons learned from the development of an abl tyrosine kinase inhibitor for chronic myelogenous leukemia," The Journal of clinical investigation, vol. 105, no. 1, pp. 3-7, 2000. 
3. L. Caldemeyer, M. Dugan, J. Edwards, and L. Akard, "Long-term side effects of tyrosine kinase inhibitors in chronic myeloid leukemia," Current hematologic malignancy reports, vol. 11, no. 2, pp. 71-79, 2016.

4. A. Piccin, W. G. Murphy, and O. P. Smith, "Circulating microparticles: pathophysiology and clinical implications," Blood reviews, vol. 21, no. 3, pp. 157-171, 2007.

5. A. Mrvar-Brečko et al., "Isolated microvesicles from peripheral blood and body fluids as observed by scanning electron microscope," Blood Cells, Molecules, and Diseases, vol. 44, no. 4, pp. 307-312, 2010.

6. J. M. Aliotta et al., "Microvesicle entry into marrow cells mediates tissue-specific changes in mRNA by direct delivery of mRNA and induction of transcription," Experimental hematology, vol. 38, no. 3, pp. 233-245, 2010.

7. B. Mytar, M. Baj-Krzyworzeka, M. Majka, D. Stankiewicz, and M. Zembala, "Human monocytes both enhance and inhibit the growth of human pancreatic cancer in SCID mice," Anticancer research, vol. 28, no. 1A, pp. 187-192, 2008.

8. H. M. Valandani, N. Amirizadeh, M. Nikougoftar, M. Safa, R. M. Khalilabadi, and A. Kazemi, "Effect of extracellular vesicles derived from mesenchymal stem cells on K-562 leukemia cell line," Iran. Red Crescent Med. J, vol. 20, p. e64991, 2018.

9. K. Al-Nedawi et al., "Intercellular transfer of the oncogenic receptor EGFRvIII by microvesicles derived from tumour cells," Nature cell biology, vol. 10, no. 5, pp. 619-624, 2008.

10. M. Ganapathi et al., "Effect of Bmi1 over-expression on gene expression in adult and embryonic murine neural stem cells," Scientific reports, vol. 8, no. 1, pp. 1-10, 2018.

11. C. D'Souza-Schorey and J. W. Clancy, "Tumor-derived microvesicles: shedding light on novel microenvironment modulators and prospective cancer biomarkers," Genes \& development, vol. 26, no. 12, pp. 1287-1299, 2012.

12. K. O’Brien et al., "Exosomes from triple-negative breast cancer cells can transfer phenotypic traits representing their cells of origin to secondary cells," European journal of cancer, vol. 49, no. 8, pp. 1845-1859, 2013.

13. K. Menck et al., "Tumor-derived microvesicles mediate human breast cancer invasion through differentially glycosylated EMMPRIN," Journal of molecular cell biology, vol. 7, no. 2, pp. 143-153, 2015.

14. E. Pluskota et al., "Expression, activation, and function of integrin aMß2 (Mac-1) on neutrophilderived microparticles," Blood, The Journal of the American Society of Hematology, vol. 112, no. 6, pp. 2327-2335, 2008.

15. C. Eken, O. Gasser, G. Zenhaeusern, I. Oehri, C. Hess, and J. A. Schifferli, "Polymorphonuclear neutrophil-derived ectosomes interfere with the maturation of monocyte-derived dendritic cells," The Journal of Immunology, vol. 180, no. 2, pp. 817-824, 2008.

16. Y.-Z. Sun, J.-S. Ruan, Z.-S. Jiang, L. Wang, and S.-M. Wang, "Extracellular vesicles: a new perspective in tumor therapy," BioMed research international, vol. 2018, 2018. 
17. U. S. Kosgodage, R. P. Trindade, P. R. Thompson, J. M. Inal, and S. Lange, "Chloramidine/bisindolylmaleimide-l-mediated inhibition of exosome and microvesicle release and enhanced efficacy of cancer chemotherapy," International journal of molecular sciences, vol. 18, no. 5, p. 1007, 2017.

18. C. Tricarico, J. Clancy, and C. D'Souza-Schorey, "Biology and biogenesis of shed microvesicles," Small GTPases, vol. 8, no. 4, pp. 220-232, 2017.

19. E. Jabbour and H. Kantarjian, "Chronic myeloid leukemia: 2018 update on diagnosis, therapy and monitoring," American journal of hematology, vol. 93, no. 3, pp. 442-459, 2018.

20. I. Baluchi, H. Anani, G. Hassanshahi, A. Fatemi, and R. M. Khalilabadi, "The effect of maslinic acid on apoptotic genes in u266 multiple myeloma cell line," Gene Reports, vol. 16, p. 100431, 2019.

21. I. Baluchi, H. Anani, A. Farsinejad, A. Fatemi, and R. Mirzaeekhalilabadi, "The Antitumoral Activity of Zataria Multiflora Methanolic Extract on Acute Promyelocytic Leukemia Cell Line; NB4," J Adv Med Biomed Res, vol. 26, no. 119, pp. 43-47, 2018.

22. X. Huang, J. Cortes, and H. Kantarjian, "Estimations of the increasing prevalence and plateau prevalence of chronic myeloid leukemia in the era of tyrosine kinase inhibitor therapy," Cancer, vol. 118, no. 12, pp. 3123-3127, 2012.

23. S. Pour, A. Farsinejad, A. Fatemi, and G. Hassanshahi, "Platelet Microparticles Accelerate Proliferation and Growth of Mesenchymal Stem Cells through Longevity-Related Genes," Archives of Iranian Medicine, vol. 24, no. 8, pp. 607-614, 2021.

24. H. Anani, I. Baluchi, A. Farsinejad, A. Fatemi, and R. M. Khalilabadi, "Zataria multiflora methanolic extract has antitumor properties on U266 multiple myeloma cell line," Gene Reports, vol. 20, p. 100655, 2020.

25. D. Di Vizio et al., "Oncosome formation in prostate cancer: association with a region of frequent chromosomal deletion in metastatic disease," Cancer research, vol. 69, no. 13, pp. 5601-5609, 2009.

26. V. Muralidharan-Chari et al., "ARF6-regulated shedding of tumor cell-derived plasma membrane microvesicles," Current Biology, vol. 19, no. 22, pp. 1875-1885, 2009.

27. V. Muralidharan-Chari, J. W. Clancy, A. Sedgwick, and C. D'Souza-Schorey, "Microvesicles: mediators of extracellular communication during cancer progression," Journal of cell science, vol. 123, no. 10, pp. 1603-1611, 2010.

28. D. Di Vizio et al., "Large oncosomes in human prostate cancer tissues and in the circulation of mice with metastatic disease," The American journal of pathology, vol. 181, no. 5, pp. 1573-1584, 2012.

29. S. Jorfi et al., "Inhibition of microvesiculation sensitizes prostate cancer cells to chemotherapy and reduces docetaxel dose required to limit tumor growth in vivo," Scientific reports, vol. 5, no. 1, pp. 1-13, 2015.

30. P. Uysal-Onganer et al., "Peptidylarginine deiminase isozyme-specific PAD2, PAD3 and PAD4 inhibitors differentially modulate extracellular vesicle signatures and cell invasion in two glioblastoma multiforme cell lines," International journal of molecular sciences, vol. 21, no. 4, p. 1495, 2020. 
31. K. Keeshan, K. Mills, T. Cotter, and S. L. McKenna, "Elevated Bcr-Abl expression levels are sufficient for a haematopoietic cell line to acquire a drug-resistant phenotype," Leukemia, vol. 15, no. 12, pp. 1823-1833, 2001.

32. T. Kawano et al., "Depsipeptide enhances imatinib mesylate-induced apoptosis of Bcr-Abl-positive cells and ectopic expression of cyclin D1, c-Myc or active MEK abrogates this effect," Anticancer research, vol. 24, no. 5A, pp. 2705-2712, 2004.

\section{Figures}

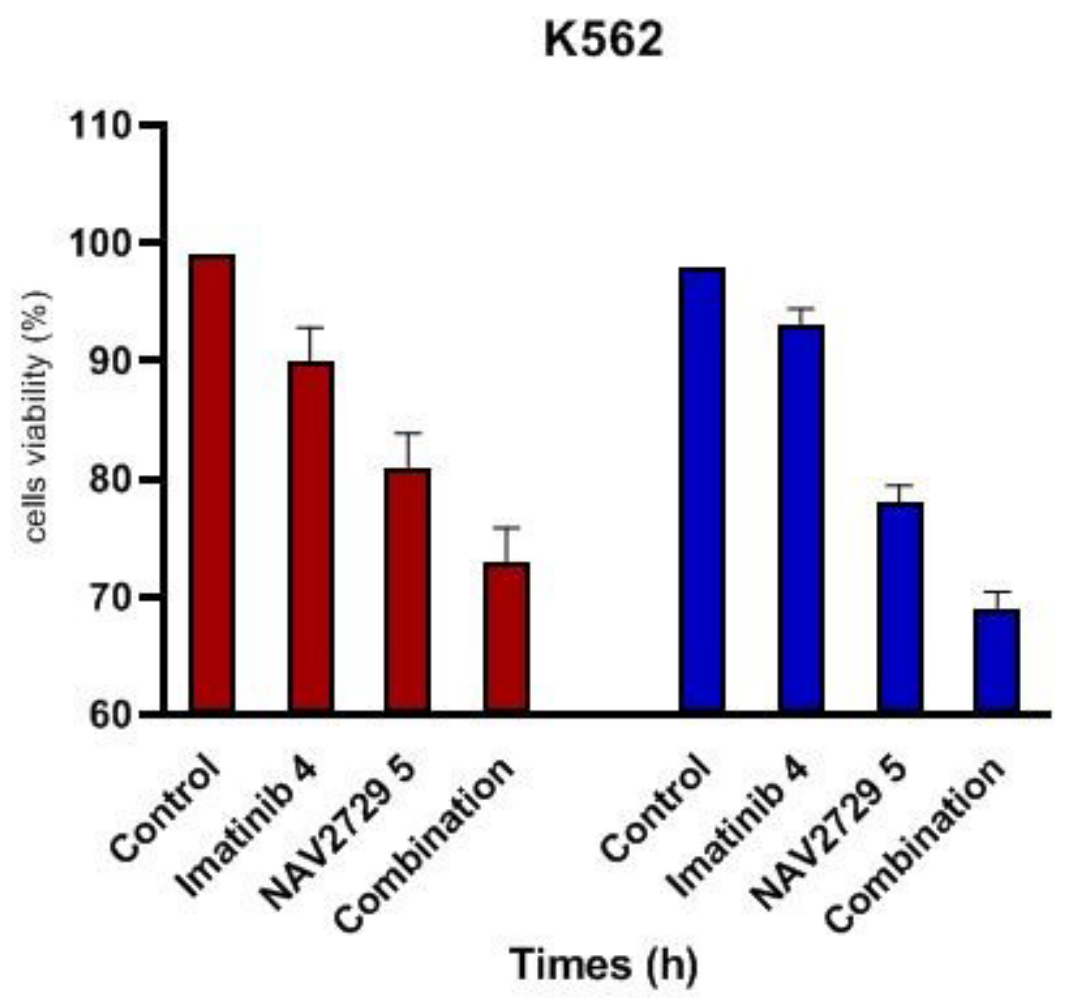

Figure 1

(a) The dose-dependent effect of NAV2729, Imatinib and the combined effect of the two drugs, on the survival of K562 cell line cells by trypan blue dye. 

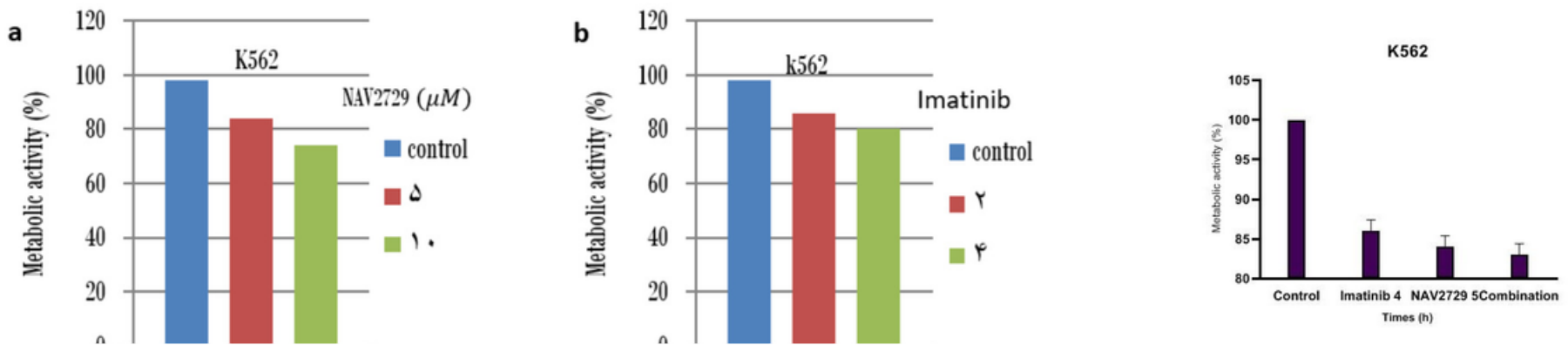

Figure 2

(a) Cellular metabolic activity in treatment with NAV2729 (b) Cellular metabolic activity in treatment with Imatinib and (c) Cellular metabolic activity in combination treatment

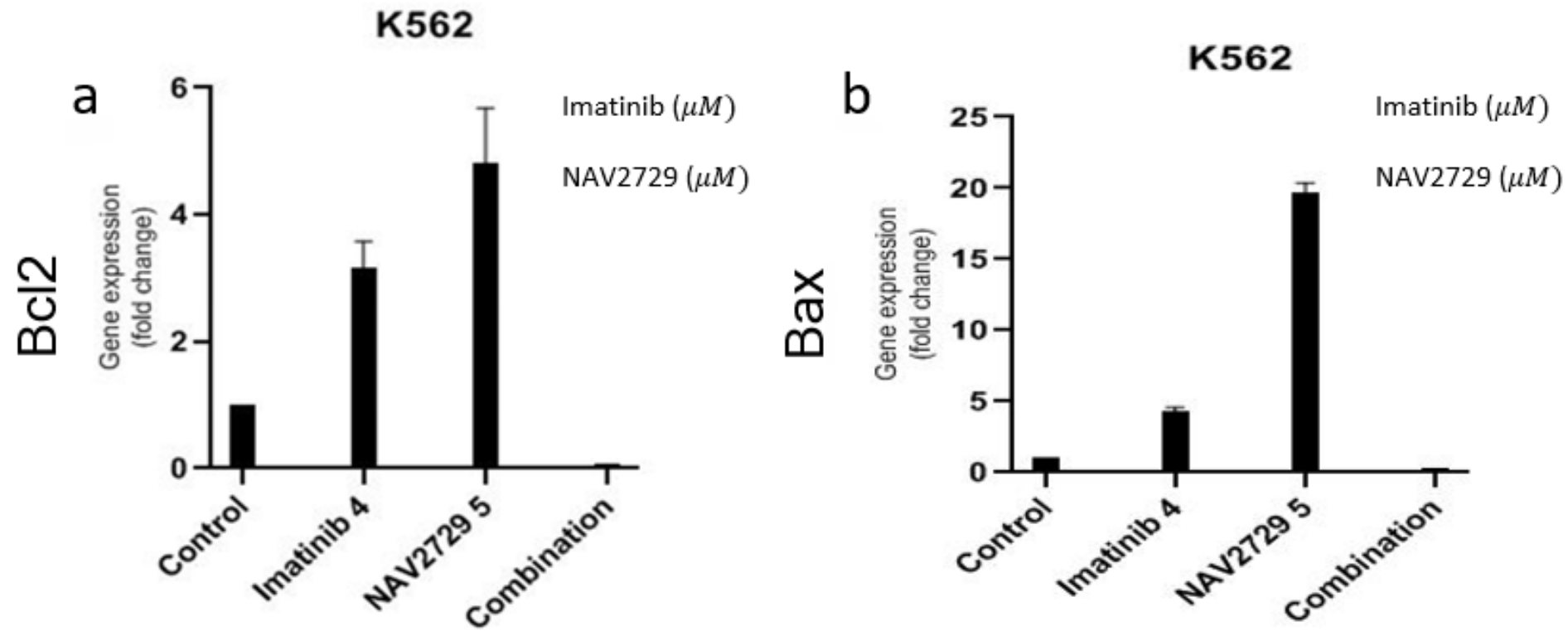

Figure 3

(a) BCL-2 gene expression in K562cells treated with separate and combined of NAV2729 and Imatinib after 48 hours. (b) BAX gene expression in K562cells treated with separate and combined of NAV2729 and Imatinib after 48 hours. 

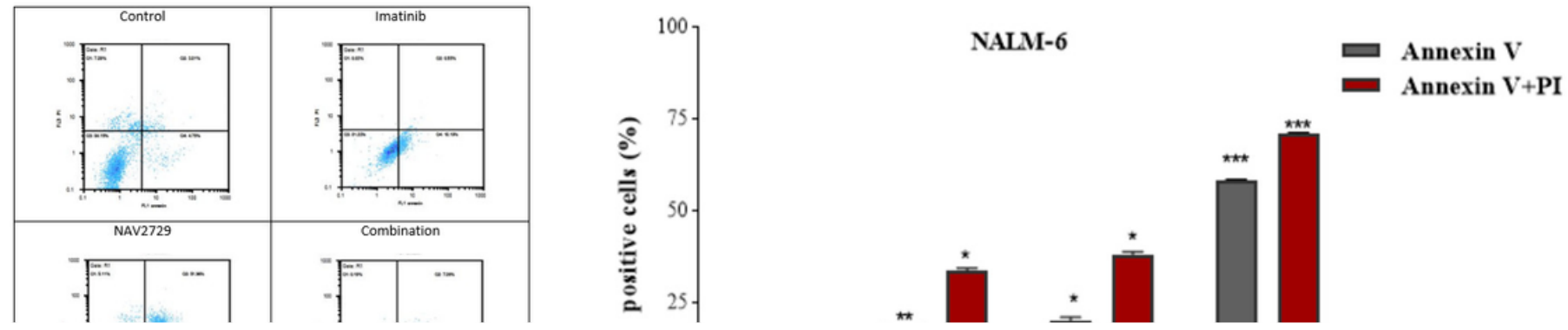

Figure 4

Apoptosis of K562cells treated with separate and combined of NAV2729 and Imatinib after 48 hours.(Pvalue * $>0.05, P V * *<0.01$, and PV ***>001/0)

\section{k562}

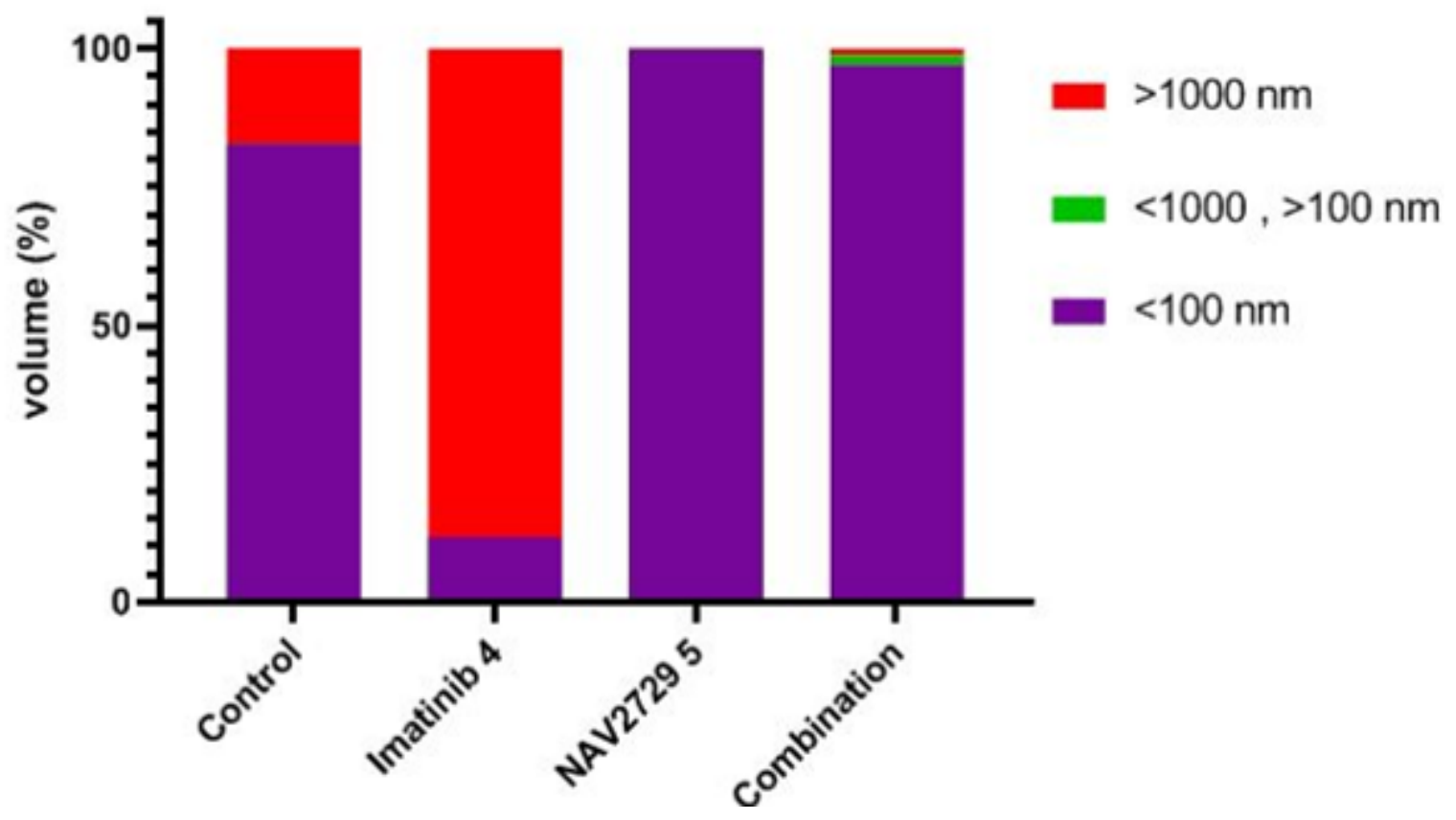

Figure 5

Size distribution of K562 derived microvesicles treated with Imatinib, NAV2729 and combination treatment, after $48 \mathrm{~h}$. 